+ und - , von denen also eine dem naturellen Corydalin mit dem
Schema + , die andere den Mesocorydalinen mit dem Schema -
+ resp. + , die vierte endlich dem 1-Corydalin mit dem Schema entsprechen müßte. Durch Vergleichen der respektiven spezifischen Drehungsvermögen mit den bei den Corydalinen beobachteten muß dann auch für letztere die Entscheidung getroffen werden können.

Diesbezügliche Versuche sind bereits im Gange.

In zweiter Linie hat Herr Haars die verschiedenen Oxydationsprodukte, welche Dobbie und Lauder aus dem Corydalin dargestellt haben, einer Nachpribung unterzogen, besonders, um festzustellen, ob die von diesen Forschern bezüglich der Entstehung der Metahemipinsäure gemachten Angaben Bestätigung fänden, da Martindale und auch Ziegenbein bei ihren Oxydationsversuchen nur die Orthosäure hatten isolieren können. Zugleich war es seine Anfgabe, die von den zuerst genannten Forschern dargestellte Corydinsäure und Corydilsäure auf die Richtigkeit der Formel zu prüfen. Dobbie und Lauder haben die Formeln dieser Säuren von einer der Perkin'schen Berberinformel nachgebildeten Konstitutionsformel des Dehydrocorydalins abgeleitet und nehmen daher in ihnen noch Parabindungen an. Daß dies nach meinen Arbeiten über das Berberin nicht zulässig ist, hat A. Werner in seinem in der Chemischen Zeitschrift. 2, 109 [1902-1903] erschienenen Referat „Unsere Kenntnisse über die Konstitution der

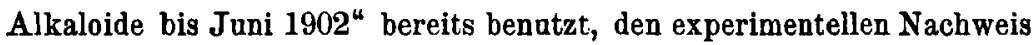
dafür zu erbringen, ist aber Herrn Haars vorbehalten geblieben. Năheres darüber ist aus der nachfolgenden Arbeit B zu ersehen.

\title{
A. Die Alkaloide der oberirdischen Teile von Corydalis cava und Corydalis solida.
}

\section{Von Dr. Otto Haars.}

Das zur Uutersuchung verwandte Kraut von Corydalis cava stammte teilweise ans dem Botanischen Garten der Universităt Marburg, wo ich es in einer Menge von $3,3 \mathrm{~kg}$ (getrocknet) sammeln lassen konnte, teilweise von der Firma Rump \& Lehners-Hannover (9 kg) und eine weitere Menge von $9 \mathrm{~kg}$ hatte ich aus der Gegend von Canth bei 
Breslau bezogen. Auch eine kleine Menge $(0,8 \mathrm{~kg})$ des Krautes von Corydalis solida wurde in den Kreis der Untersuchungen eingezogen.

Beide Pflanzen blithen im April und Mai und es sind die Kränter zur Zeit der Blüte gesammelt worden; bei dem aus Hannover bezogenen schien jedoch, soweit sich dies an der getrockneten und zerkleinerten Droge feststellen ließ bereits teilweise die Samenreife eingetreten za sein.

Bei einem Vorversuch mit $500 \mathrm{~g}$ erprobte ich die günstigsten Bedingangen, unter denen sich das Kraut verarbeiten lassen würde und fand die Methode, die ursprünglich von Alexander Ehrenberg ${ }^{1}$ ) bei der Verarbeitung der Knollen von Corydalis cava angewendet und später von Gadamer ${ }^{2}$ ) verbessert wurde, auch fïr das Kraut anwendbar.

Es wurden hiernach $500 \mathrm{~g}$ der zerkleinerten Droge in einem gläsernen Perkolator mit Alkohol erschöpft, der Alkohol abdestilliert, soda $B$ ein dickes, dunkelgrünes Extrakt zurückblieb. Dasselbe wurde nach und nach mit etwa der zehnfachen Menge essigsäurehaltigem Wasser versetzt. Es schieden sich nach mehrtägigem Stehen die Fette, Harze, das Chlorophyll and andere der weiteren Verarbeitung hinderliche Stoffe ab. Nachdem dieselben durch Filtration, von der wässerigen Lösung getrennt worden und nochmals mit essigsäurehaltigem Wasser ausgezogen waren, wurde die Alkaloidlösung im Scheidetrichter ammoniakalisch gemacht und mit Aether zweimal und darauf mit Chloroform ausgeschüttelt. Der Aether wurde abdestilliert; nach karzer Zeit schieden sich aus der atherischen Lösung kleine sehr gut ausgebildete Krystalle ab. Aus der Chloroformausschlittelung konnte eine Krystallisation nicht erzielt werden. Die aut ein sehr kleines Volumen eingeengten Aether- und Chloroformausschüttelnngen worden vereinigt, zunächst mit etwas Alkohol, dann mit salzsäurehaltigem Wasser aufgenommen; wieder ammoniakalisch gemacht und mit Aether ausgeschüttelt. Es gelang so nach dem Abdestillieren des Aethers noch weitere Krystallisationen zu erhalten.

Aus den angewandten $500 \mathrm{~g}$ Kraut erhielt ich eine Ausbeate von 3,05 g Rohalkaloiden, was einem Gehalt von $0,6 \%$ entspricht. Zur Reinigung wurden die Alkaloide nochmals in salzsăurehaltigem Wasser gelðst, die Lơsung mit Ammoniak im Ueberschuß versetzt und mit Aether ausgeschüttelt. Ich erhielt nach dem Abdestillieren des Aethers gelblich gefärbte Krystalle vom Schmp. 195-196 ${ }^{\circ}$. Nach dem Umkrystallisieren wurden dieselben fast farblos, der Schmelzpunkt stieg auf $199^{\circ}$.

1) Annal. d. Chem. 277, S. 4.

2) Arch. d. Pharm. 240, S. 21. 
0. Haars: Alkaloide des Krants von Corydalis.

Zunăchst prüfte ich mit den ïblichen Alkaloidreagentien und konnte folgendes Verhalten feststellen:

\begin{tabular}{c|c|c|c|c}
\hline Konz. $\mathrm{H}_{2} \mathrm{SO}_{4}$ & Konz. HNO 8 & $\begin{array}{c}\text { Erdmann's } \\
\text { Reagens }\end{array}$ & $\begin{array}{c}\text { Fröhde's } \\
\text { Reagens }\end{array}$ & $\begin{array}{c}\text { Vanadin- } \\
\text { schwefelsăure }\end{array}$ \\
\hline \hline $\begin{array}{c}\text { erst farblos } \\
\text { dann schwach } \\
\text { orange beim } \\
\text { Erwarmen } \\
\text { violett }\end{array}$ & rotbrann. & blanviolett. & $\begin{array}{c}\text { dunkelblau, } \\
\text { später } \\
\text { schwarz. }\end{array}$ & $\begin{array}{c}\text { tief } \\
\text { dunkelblau. }\end{array}$ \\
\end{tabular}

Die Elementaranalyse lieferte folgende Werte:

0,2075 g Substanz gaben 0,5322 g $\mathrm{CO}_{2}$ und $0,114 \mathrm{~g} \mathrm{H}_{2} \mathrm{O}$.

0,1986 n $, \quad 7,0 \mathrm{ccm} N$ bei 140 und 765 mm Druck.

Nach diesen Daten und dem beschriebenen Verhalten gegen Alkaloidreagentien, ferner nach dem ermittelten Schmelzpunkt konnte es keinem Zweifel unterliegen, daf hier das von Freund und Jesephi zuerst beschriebene und von ihnen Bulbocapnin benannte Alkaloid vorlag, dem die Formel $\mathrm{C}_{19} \mathrm{H}_{19} \mathrm{NO}_{4}$ zukommt.

$$
\begin{array}{cc}
\text { Berechnet für } \mathrm{C}_{19} \mathrm{H}_{19} \mathrm{NO}_{4}: & \text { Gefunden: } \\
\mathrm{C}=70,1 & 70,0 \% \\
\mathrm{H}=5,8 & 6,0 \% \\
\mathrm{~N}=4,3 & 4,2_{\eta} .
\end{array}
$$

Zur weiteren Charakterisierung stellte ich noch das salzsaure Salz dar, das sich durch seine große Krystallisationsfahigkeit auszeichnet. Es bildet lange, weiße, seidenglänzènde Nadeln, die ich erhielt beim Versetzen einer alkoholischen Auflösung der Base mit salzsäurehaltigem Wasser bis zur schwach sauren Reaktion.

Die Analyse des Salzes führte zu der Formel: $\mathrm{C}_{19} \mathrm{H}_{19} \mathrm{NO}_{4} \cdot \mathrm{HCl}$.

$0,3106 \mathrm{~g}$ lieferten 0,1254 g Ag Cl.

Berechnet für $\mathrm{C}_{10} \mathrm{H}_{10} \mathrm{NO}_{4} \mathrm{HCl}$ :

$\mathrm{Cl}=\mathbf{9 , 8}$

Gefunden:

$9,9 \%$

Aus dem Kraut von Corydalis solida kunnte ich in gleicher Weise Bulbocapnin isolieren.

Um entscheiden zu können, ob außer Bulbocapnin noch andere Alkaloide im Kraut von Corydalis cava enthalten seien, mußten naturgemäß größere Mengen zur Verarbeitung gelangen, da nur dann 
solche Alkaloide (ev. auch das Protopin) aufgefunden werden konnten, die nur in geringerer Menge im Kraut vorkommen. In der Tat zeigte sich, als nach der oben beschriebenen Methode je $9 \mathrm{~kg}$, bezogen von Rump \& Lehners resp. bei Canth gesammelt, verarbeitet wurden, daß sich, nachdem schon reichliche Mengen Bulbocapnin aus den ätherischen Lösungen auskrystallisiert und gesammelt worden waren, eine kleine Menge eines Alkaloides abschied, das durch seine Unlöslichkeit in Alkohol ausgezeichnet war. Ich wurde auf dasselbe aufmerksam, als ich die dritte Krystallisation aus der ätherischen Lösung aus Alkohol umzakrystallisieren versuchte. Es hinterblieben hier grünlich braun gefärbte Krystalle, die auch durch anhaltendes Kochen nicht in Alkohol gelöst werden konnten. Ihr Schmelzpankt lag bei 223-224 ${ }^{\circ}$. In erwärmtem Chloroform war das Alkaloid ziemlich leicht löslich, sodaß ich es aus einer Chloroform-Alkohol-Mischnng umkrystallisieren konnte. Nach mehrfachem Umkrystallisieren waren die ausgeschiedenen Krystalle gelblichweiß, der Schmelzpunkt war auf $230^{\circ}$ gestiegen.

Durch wiederholtes Ausziehen des Harzes, der fett- und chlorophyllhaltigen Bestandteile, die sich beim Anfnehmen des ursprünglichen Extraktes mit essigsăurehaltigem Wasser abgeschieden hatten, mit salzsäurehaltigem Wasser, gelang es noch eine weitere geringe Menge des bei $230^{\circ}$ schmelzenden Alkaloides zu erhalten. Die salzsauren Auszüge wurden mit Ammoniak im Ueberschuß versetzt, mit Aether ausgeschüttelt, der Aether abdestilliert und der Rückstand mit salzsäurehaltigem Wasser aufgenommen und in überschüssige $5 \%$ ige Natronlauge eingetragen. Das Bulbocapnin, als alkalilöslich, verblieb in der Lösung, während ein Teil als grauweißer, amorpher Niederschlag ausfiel. Dieser Niederschlag wurde gesammelt, wieder in salzsäurehaltigem Wasser gelöst, durch Ammoniak abgeschieden und mit Aether aufgenommen. Es hinterblieben so nach dem Verdunsten des Aethers kleine, gelblich weiße Krystalle, die nach dem Umkrystallisieren aus Chloroformalkohol den Schmp. $230^{\circ}$ zeigten.

\section{Alkaloid $\mathrm{C}_{21} \mathrm{H}_{21} \mathrm{NO}_{8}$.}

Das bei $230^{\circ}$ schmelzende Alkaloid ist in ätzenden Alkalien unlöslich, enthält also keine Phenolhydroxylgruppen. Im Gegensatz zu allen anderen Corydalisalkaloiden dreht dieses Alkaloid den polarisierten Lichtstrabl nach links. Der Schmelzpunkt, die Linksdrehung des polarisierten Lichtstrahls weisen auf die Möglichkeit einer Identităt mit Morphin hin, gegen die andererseits die Unlöslichkeit in ätzenden Alkalien sprach. Einige charakteristische Reaktionen des 
Morphins zog ich zum Vergleich heran; sie tiberzeugten mich, daß Morphin nicht vorlag.

Wird in die Lösung des Morphins in konzentrierter Schwefelsåure eine geringe Menge basisches Wismutnitrat eingetragen, so tritt sofort eine rote bis schwarzbraune Fr̈rbung ein; das bei $230^{\circ}$ schmelzende Alkaloid zeigte unter gleichen Bedingungen eine blauschwarze' Färbang.

Fröhde's Reagens liefert mit Morphin eine charakteristische, violette Färbung, die sehr bald in Blau übergeht; das neue Alkaloid zeigte im Anfang eine gríne Farbe, die später jedoch auch in Blau iiberging.

Das Verhalten gegen die üblichen Alkaloidreagentien war folgendes:

\begin{tabular}{|c|c|c|c|c|}
\hline Konz. $\mathrm{H}_{2} \mathrm{SO}_{4}$ & Konz. $\mathrm{HNO}_{6}$ & $\begin{array}{c}\text { Erdmann's } \\
\text { Reagens }\end{array}$ & $\begin{array}{l}\text { Fröhde's } \\
\text { Reagens }\end{array}$ & $\begin{array}{c}\text { Vanadin- } \\
\text { schwefelsäure }\end{array}$ \\
\hline farblos. & $\begin{array}{l}\text { erst farblos, } \\
\text { dann intensiv } \\
\text { gelb. }\end{array}$ & $\begin{array}{l}\text { gelb, hell- } \\
\text { grün, oliv- } \\
\text { farben, } \\
\text { zuletzt } \\
\text { schmutzig } \\
\text { rötlich. }\end{array}$ & $\begin{array}{l}\text { grün, blau- } \\
\text { schwarz, } \\
\text { violett. }\end{array}$ & $\begin{array}{l}\text { erst grün, } \\
\text { dann schiefer- } \\
\text { farben, blau } \\
\text { mit rötlichem } \\
\text { Ton, vom } \\
\text { Rande her } \\
\text { rot. }\end{array}$ \\
\hline
\end{tabular}

Da leider nur eine geringe Menge des Alkaloids zur Verfügung stand - aus $18 \mathrm{~kg}$ trockenen Krautes konnte ich nur $1,5 \mathrm{~g}$ isolieren - so konnte von einer definitiven Aufstellung einer Formel nicht die Rede sein. Die Ergebnisse der Elementaranalysen stehen mit der Formel $\mathrm{C}_{21} \mathrm{H}_{21} \mathrm{NO}_{8}$ jedenfalls nicht in Widerspruch:

$0,2068 \mathrm{~g}$ verloren im Vakuumexsiccator über Schwefelsäure getrocknet nichts an Gewicht.

1. $0,2035 \mathrm{~g}$ gaben $0,0974 \mathrm{~g} \mathrm{H}_{2} \mathrm{O}$ und $0,4518 \mathrm{~g} \mathrm{CO}_{2}$.

2. $0,1988 n n 0,0912 n, n 0,4394 n n$.

3. $0,1955 n, 5,5 \mathrm{ccm} \mathrm{N}$ bei $16^{\circ}$ und $756 \mathrm{~mm}$ Druck.

\begin{tabular}{cccc} 
Berechnet für & \multicolumn{3}{c}{ Gefunden: } \\
$\mathrm{C}_{92} \mathrm{H}_{21} \mathrm{NO}_{8}:$ & 1. & 2. & $\mathbf{3}$. \\
$\mathbf{C}=60,7$ & 60,6 & $60,3 \%$ & - \\
$\mathrm{H}=5,1$ & 5,3 & $5,1 n$ & - \\
$\mathrm{N}=\mathbf{3 , 4}$ & - & - & $\mathbf{3 , 3} \%$.
\end{tabular}

Das von Hesse aus Papaver Rhoeas und Papaver somniferum isolierte Rhoeadin zeigt im Schmp. (232 $)$ und der Molekularformel $\left(\mathrm{C}_{21} \mathrm{H}_{21} \mathrm{NO}_{6}\right)$ eine gewisse Aehnlichkeit mit dem hier beschriebenen Alkaloid, ist aber sicher nicht damit identisch, wie schon aus dem 
Verhalten gegen Sänren hervorgeht. Man muß daher in dem Alkaloid ein bisher noch nicht bekanntes erblicken. Einen Namen habe ich demselben nicht gegeben, da es vorzuziehen sein wird, erst noch weiteres Material herbeizuschaffen, um feststellen zu können, zu welchen bekannten Alkaloiden das nea aufgefundene in näherer Beziehung steht und dann erst einen geeigneten Namen zu wăhlen.

Bestimmungdes spezifischen Drehungsvermögens.

Sämtliche bisher aus Corydalis cava isolierten Alkaloide drehen, soweit sie optisch aktiv sind, das polarisierte Licht nach rechts, wie ans folgender Zusammenstellung ersichtlich ist.

$\begin{array}{ll}\text { Corydalin } & \left.[\alpha] \mathrm{D}=+300,1^{01}\right) \\ \text { Corybulbin } & \left.\left.[\alpha]_{\mathrm{D}}^{20}=+311,002\right)+309,502\right) \\ \text { Isocorybulbin } & {[\alpha]_{\mathrm{D}}^{20}=+299,80} \\ \text { Corycarin } & \text { optisch inaktiv }) \\ \text { Corycavamin } & \left.[\alpha]_{\mathrm{D}}^{20}=+166,6^{408}\right) \\ \text { Bulbocapnin } & \left.[\alpha]_{\mathrm{D}}^{20}=+237,1^{00}\right) \\ \text { Corydin } & \left.[\alpha]_{\mathrm{D}}^{20}=+204,33^{07}\right) \\ \text { Corytuberin } & \left.[\alpha]_{\mathrm{D}}^{20}=+282,6508\right)\end{array}$

Die Linksdrehung ist also für dieses neue Alkaloid besonders charakteristisch.

$0,2607 \mathrm{~g}$ löste ich in Pyknometer bei $20^{\circ}$ in Chloroform, fullte $\mathrm{zu}$ $25 \mathrm{ccm}$ auf und bestimmte das spezifische Gewicht der Losung $(\alpha=1,4824)$. Im 220 mm-Robr betrug im Laurent'schen Halbschattenapparat

$$
[a]_{\mathrm{D}}^{20}=-3,67^{\circ} \text { (Mittel von } 6 \text { Ablesungen). }
$$

Daraus berechnet sich $[a]_{D}^{20}=-112,80$. Für Morphin wurde von Hesse? $[\alpha]_{\mathrm{D}}=-98,41^{\circ}$ ermittelt.

1) Freund und Josephi, Annal. d. Chem. 277, S. 7.

2) Dobbie and Lauder, Transact. of the Chem. Soc. Corydaline IV.

B) Gadamer und Bruns, Arch. d. Pharm. 230, S. 41.

4) Wagner, Inaug.-Diss., Marburg 1901.

b) Gadamer, Arch. d. Pharm. 240, S. 85.

-) Gadamer, Arch. d. Pharm. 240, S. 85.

7) Gadamer, Arch. d. Pharm. 240, S. 99.

8) Wagner, Inaug.-Diss. Marburg 1901.

9) Annal. d. Chem. 176, 189. 
Methoxylbestimmung. Die nach dem Verfahren von Zeisel ") im Apparat nach Benedikt und Grüßner ${ }^{2}$ ) ausgefuhrte Methoxylbestimmung verlief negativ. Nach einstündigem Erhitzen zeigte die vorgelegte Silbernitratlösung nicht die geringste Trübung. Methoxylgruppen sind demnach in dem Alkaloid nicht enthalten.

Das Goldsalz des Alkaloides entsteht als flockiger, fleischfarbener Niederschlag, wenn man die salzsaure Lösung des Alkaloides mit Goldchlorid versetzt. Dasselbe ist schwer löslich in Wasser, leichter in Alkohol, beim Erwärmen backt es harzartig zusammen und nimmt dabei eine rote Farbe an. Das Goldsalz läßt sich weder aus Wasser noch aus Alkohol umkrystallisieren, es konnte daher eine A nalyse nicht ausgeführt werden.

Das Platinsalz wurde als fast weißer flockiger Niederschlag erhalten beim Versetzen einer Lösung des Alkaloides in salzsäurehaltigem Wasser mit einer filtrierten Platinchloridlosung. Beim Erwärmen ist es in Wasser löslich, scheidet sich jedoch beim Erkalten wieder flockig aus. Ein gleiches Verhalten zeigt eine alkoholische Lösung. Der Schmelzpunkt des pulverförmigen, gelblich-weißen Platinsalzes lag bei $214^{\circ}$.

$0,1790 \mathrm{~g}$ über Schwefelsäure im Vakuum getrocknet hinterließen beim Glühen $0,0290 \mathrm{~g}$ Pt.

Berechnet für $\left(\mathrm{C}_{21} \mathrm{H}_{21} \mathrm{NO}_{8}\right)_{2} \mathrm{H}_{2} \mathrm{PtCl}_{6}$ :

$$
\mathrm{Pt}=15,7
$$

Gefunden:

$16,1 \%$

Auf eine weitere Darstellung von Salzen mußte ich wegen der Spärlichkeit des Materials verzichten.

1) Monatshefte f. Chem. 1885, 989.

2) Chem.-Ztg. 1889, 872.

(Fortsetzung folgt.) 


\section{Alkaloid $\mathrm{C}_{21} \mathrm{H}_{28} \mathrm{NO}_{7}$.}

Bei der weiteren Verarbeitung der alkoholischen Mutterlaugen, ans denen also Bulbocapnin und jenes eben beschriebene bei $280^{\circ}$ schmelzende Alkaloid isoliert waren, konnte ich die Anwesenheit noch eines anderen Alkaloides feststellen, das bisher auch nicht in den Knollen von Corydalis cava gefunden worden ist.

Die Laugen wurden mit Salzsäure sorgfältig neutralisiert, der Alkohol verdunstet and jetzt die Lösung mit Rhodanammonium gefält bis auf weiteren Zusatz kein Niederschlag mehr entstand. Der Niederschlag war harzig, schmierig und setzte sich sehr bald fest am Boden und Wandungen des Gefảes an, wăhrend die aberstehende Flüssigkeit sich klärte. Sie wurde abgegossen und durch Alkohol ersetzt. Nach Verlauf von mehreren Tagen warde ein Teil des Niederschlages krystallinisch, wahrend ein anderer Teil in Losung gegangen war. Diese krystallinischen Rhodanbasen wurden gesammelt, mit konzentrierter alkoholischer Ammoniakflüssigkeit zerlegt, mit Aether ansgeschüttelt. Nach dem Abdestillieren des Aethers and dem Umkrystallisieren des Räckstandes aus Alkohol ergab sich durch Bestimmung des Schmelzpunktes, der zu $19^{\circ}$ gefunden warde, daB dieser Teil noch aus Bulbocapnin bestand.

Die amorphen Rhodanbasen, die in Alkohol löslich waren, wurden nach möglichster Entfernung des Alkohols durch Ammoniak zerlegt und mit Aether ausgeschüttelt. Die ätherische Alkaloidlösung wurde mit salzsäurehaltigem Wasser auggeschüttelt, sodaB die Alkaloide aus dem Aether jetzt als salzsaure Salze in die wässerige Flüssigkeit gingen. Durch Zusatz von Ammoniak wurden die freien Basen wieder abgeschieden und mit Aether aufgenommen. Bei der weiteren Verarbeitung hat sich das Verfahren der partiellen Sättigung als sehr brauchbar erwiesen. Durch einen Vorversuch hatte ich gefunden, $\mathrm{daB}$ in $10 \mathrm{~g}$ der ätherischen Lösung $0,6 \mathrm{~g}$ Alkaloid enthalten waren. In 160,0 vorhandener waren demnach 9,6 g Alkaloid. Als mittleres Molekulargewicht der Alkaloide nahm ich 360 an. Daraus berechnet sich, daB $26,6 \mathrm{ccm} \mathrm{n} / 1$ Salzsăure zar Săttigung genügen würden. Ich schüttelte nun die Gesamtmenge der ătherischen Lösung mit je $5 \mathrm{ccm}$ n/1 Salzsäure sechsmal ans, trennte sorgfältigst und überlieB die salzsauren Lösungen der Krystallisation. Die erste bis dritte Fraktion hatte eine dunkelgrüne Farbe, die allmählich in den letzten Fraktionen in eine dunkelrotbraune überging. Aus Fraktion 1-3 schieden sich sehr bald rosettenförmig angeordnete, seidenglänzende Krystalle aus, die, wie beim Zerlegen durch Ammoniak und Ausschütteln mit Aether festgestellt wurde, aus salzsaurem Bulbocapnin bestanden. Die freie Base schmolz bei 1990 .

Beim Zerlegen der Fraktionen fünf and sechs erhielt ich eine Krystalli-. sation, welche den Schmp. $135^{\circ}$ zeigte, und ich glaubte annehmen zu dürten, $\mathrm{daB}$ hier das in den Knollen so reichlich vorkommende Corydalin (Schmp. 134,50) vorläge. Diese Annahme erwies sich jedoch als irrig. Nach dem Umkrystallisieren aus Alkohol, in dem die Base sebr leicht löslich ist, stieg der Schmelzpunkt auf 137,50. Aus Alkohol krystallisiert sie in durchsichtigen 
stark lichtbrechenden, rechteckigen Prismen, die hăufig zu Rosetten angeordnet sind. Die Ausbeute betrug $0,5 \mathrm{~g}$.

Dann dachte ich an die Möglichkeit einer Identität mit dem Chelidonin, dessen Schmelzpunkt bei $136^{\circ}$ liegt, zumal ich fand, daß es die Eigenschaft der Triboluminescenz mit dem Chelidonin gemeinsam hat, die von J. O. Schlotterbeck und H. C. Watkins ') bei jener Base beobachtet wurde. Beim Reiben der Krystalle mit einem Glasstab an den Wandungen eines Reagensglases konnte ich im dunklen Zimmer deutlich das Umhersprühen vieler blauweißer Fünkchen beobachten. Allein die Bestimmung des spezifischen Drehungsvermögens, die Elementaranalyse, ferner die Gegenwart zweier Methoxylgruppen neben einer Methylimidgruppe in dem vorliegenden Alkaloid bewiesen die Verschiedenheit vom Chelidonin auf das dentlichste.

Den üblichen Alkaloidreagentien gegenäber zeigte es folgendes Verhalten:

\begin{tabular}{|c|c|c|c|c|}
\hline Konz. $\mathrm{H}_{2} \mathrm{SO}_{4}$ & Konz. $\mathrm{HNO}_{3}$ & $\begin{array}{l}\text { Fröhde's } \\
\text { Reagens }\end{array}$ & $\begin{array}{c}\text { Erdmann's } \\
\text { Reagens }\end{array}$ & $\begin{array}{c}\text { Vanadin- } \\
\text { schwefelsäure }\end{array}$ \\
\hline $\begin{array}{l}\text { bräunlich, } \\
\text { sehr bald } \\
\text { kirschrot, } \\
\text { zuletzt } \\
\text { violett. }\end{array}$ & rotbraun. & $\begin{array}{l}\text { kornblum- } \\
\text { blau, das } \\
\text { allnablich } \\
\text { eine } \\
\text { schmutzige } \\
\text { Farbe } \\
\text { annimmt. }\end{array}$ & $\begin{array}{l}\text { dunkelblau, } \\
\text { grün, oliven- } \\
\text { farben, vom } \\
\text { Rand her } \\
\text { rötlich. }\end{array}$ & $\begin{array}{l}\text { hellblau, } \\
\text { blaugrün, } \\
\text { grün, zuletzt } \\
\text { schmutzig } \\
\text { grün. }\end{array}$ \\
\hline
\end{tabular}

Bestimmung des spezifischen Drehungsverm ogens.

$0,4425 \mathrm{~g}$ löste ich im Pyknometer in Alkohol und füllte bei $20^{\circ} \mathrm{zu} 25 \mathrm{ccm}$ auf. Die Drehung betrug: $[\alpha]_{\mathrm{D}}^{20}=+3,770$.

Daraus berechnet sich: $[\alpha]_{\mathrm{D}}^{20}=+96,8^{\circ}$.

Zum Vergleich zog ich das optische Drehungsvermogen des Chelidonins heran, das ja in Bezug auf den Schmelzpunkt und die Eigenschaft der Triboluminescenz mit dem vorliegenden Alkaloid große Aehnlichkeit zeigte. Ich fand dafür folgenden Wert:

$0,4425 \mathrm{~g}$ Chelidonin wurden in $25 \mathrm{ccm}$ Alkohol gelöst.

Die Drehung betrug: $[\alpha]_{\mathrm{D}}^{20}=+4,50^{\circ}$.

Folglich: $[a]_{\mathrm{D}}^{20}=+115,00$.

Dieser Wert stimmt mit dem von Wintgen ${ }^{2}$ ) für das Chelidonin angegebenen

$$
[a]_{D}^{20}=+115,24^{0}
$$

1) Ber. 35, S. 10 a. 11.

2) Arch. d. Pharm. 1901, 446. 
gut itberein und zeigt zugleich die Verschiedenheit des bei $137,5^{\circ}$ schmelzenden Alkaloides vom Chelidonin in Bezug anf das spezifische Drehungsvermogen.

Die Elementaranalyse lieferte folgende Werte:

1. $0,1823 \mathrm{~g}$ gaben $0,1047 \mathrm{~g} \mathrm{H}_{2} \mathrm{O}$ und $0,4190 \mathrm{~g} \mathrm{CO}_{2}$.

2. $0,1840 \mathrm{~g}$ gaben $6,0 \mathrm{ccm} \mathrm{N}$ bei $15^{\circ}$ und $748 \mathrm{~mm}$ Druck.

Gefunden:

$$
\begin{aligned}
& \mathrm{C}=62,7 \% \\
& \mathrm{H}=6,4{ }^{\prime} \\
& \mathrm{N}=3,7 n .
\end{aligned}
$$

Die Werte passen zu der Formel

$$
\begin{array}{ccc}
\mathrm{C}_{21} \mathrm{H}_{28} \mathrm{NO}_{7}: & \text { oder } & \mathrm{C}_{21} \mathrm{H}_{25} \mathrm{NO}_{7}: \\
\mathrm{C}=62,8 & & 62,5 \% \\
\mathrm{H}=5,8 & 6,2 \% \\
\mathrm{~N}=3,5 & 3,5 \% .
\end{array}
$$

Methoxylbestimmung nach Zeisel. Die nach Zeisel im Apparat nach Benedikt und Grüßner ausgeführte Methoxylbestimmung führte zu keinem Resultat, welches mit den obigen Analysen in Einklang zu bringen gewesen wäre.

0,1210 g gaben 0,1816 g Ag J.

Berechnet für

$$
\begin{gathered}
\mathrm{C}_{21} \mathrm{H}_{25} \mathrm{NO}_{7}: \\
2 \mathrm{OCH}_{8}=15,4
\end{gathered}
$$$$
3 \mathrm{OCH}_{8}=23,1
$$

$\mathrm{C}_{21} \mathrm{H}_{28} \mathrm{NO}_{7}$ :

$15,5 \%$

23,2 ,
Gefunden:

$19,8 \%$.

Daß die Methode von Zeisel in gewissen Fällen im Stich läbt, ist von verschiedenen Autoren beobachtet worden, so hat Pommerehne ${ }^{1}$ ) festgestellt, gelegentlich seiner Untersuchungen uber das Damascenin, daß nach dem Verfahren von $Z$ eisel je nach der Dauer des Erhitzens mit starker Jodwasserstoffsäure verschiedene Werte für Methoxyl erhalten werden. Während er in seiner ersten Veröffentlichung für das Damascenin swei Methoxylgruppen annimmt, kommt er später zu dem Schluß, daß nur eine Methoxylgruppe vorliegt. Im ersten Falle hatte er 4 Stunden, im letzteren 1//2 Stunden erhitzt. Dieser Punkt ist neuerdings von $\mathrm{O} . \mathrm{Ke} l 1 \mathrm{er}^{2}$ ) dahin klargestellt worden, $\mathrm{daB}$ im Damascenin eine $\mathrm{OCH}_{8}$-Gruppe and eine $\mathrm{NCH}_{8}$-Gruppe enthalten ist. Aus den Angaben von Pommerehne geht also hervor, $\mathrm{da} \beta$ eine teilweise $\mathrm{Abspaltung}$ der $\mathrm{NCH}_{8}$-Gruppe bereits bei längerem Erhitzen nach der Zeisel'schen Methode erfolgt.

1) Arch. d. Pharm. 237, 480.

2) Arch. d. Pharm. 238, 546. Apoth-Ztg. 1903, 684. 
Beim Oxyacanthin hat $\mathrm{Pommerehne} \mathrm{gleiche} \mathrm{Beobachtungen}$ gemacht; er erhielt hier nach dem Zeisel'schen Verfahren Werte, die $\mathrm{zwischen}$ den für 1 und 2 Methoxylgruppen berechneten lagen.

Goldschmiedt ${ }^{1}$ ) und Hönigschmid ${ }^{2}$ ) berichteten von dem Methylbetain der Chinolinsäure und Papaverinsäure, daß ein bedeutender Teil des an Stickstoff gebundenen Methyls durch siedende Jodwasserstoffsăure nach dem Zeisel'schen Verfahren abgespalten wird.

Diese Tatsachen legten daher in meinem Falle die Annahme nahe, daß in dem Alkaloid neben zwei Methoxylgruppen eine Methylimidgruppe vorliegen möchte. Herzig und $\mathrm{Meyer}^{8}$ ) haben eine Methode ausgearbeitet, welche unter Anlehnung an die Zeisel'sche Methoxylbestimmung eine quantitative Bestimmung der Methylimidgruppen bei den Methoxylgruppen ermöglicht. Die nach jenen Angaben ausgef ührte Bestimmung lieterte folgende Werte:

0,1630 g gaben bei der Zersetzung im Glyzerinbade 0,1972 g Ag J.

Berechnet für 2 Methoxylgruppen: Gefunden: in $\mathrm{C}_{21} \mathrm{H}_{25} \mathrm{NO}_{7} \quad$ in $\mathrm{C}_{21} \mathrm{H}_{28} \mathrm{NO}_{7}$ $\mathrm{OCH}_{8}=15,36 \quad 15,46 \quad 15,97 \%$.

Bei der Zersetzung im Sandbade wurden aus der vorgelegten Silbernitratlösung noch 0,1048 g Ag J abgeschieden.

Berechnet für $1 \mathrm{CH}_{8}$-Gruppe: Gefunden:

$\begin{array}{ccc}\text { in } \mathrm{C}_{21} \mathrm{H}_{25} \mathrm{NO}_{7} & \text { in } \mathrm{C}_{21} \mathrm{H}_{23} \mathrm{NO}_{7} \\ \mathrm{CH}_{8}=3,72 & 3,73 & 4,1 \% \text {. }\end{array}$

Das vorliegende Alkaloid enthalt also zwei Methoxyl- und eine Methylimidgruppe.

In letzter Zeit hat G. Heyl ${ }^{4}$ aus Dicentra formosa ein Alkaloid isoliert, welches in Bezug auf den Schmelzpunkt $\left(142^{\circ}\right)$ und die auch

1) Arch. d. Pharm. 233, 152.

2) Ber. 1903, 1853.

$\left.{ }^{8}\right)$ Ehe ich an die mir neue Methode der Bestimmung der Methylimidgruppe heranging und dieselbe bei dem mir in geringer Menge zur Verfügung stehenden Material anwandte, studierte ich sie zunăchst an anderen leichter zugänglichen Alkaloiden: dem Bulbocapnin und Chelidonin. Beide sind von Herzig und Mejer schon untersucht; meine Resultate dienen also nur zur Bestătigung:

Bulbocapnin: 0,1935 g Substanz gaben 0,1358 g 0-Ag J u. 0,139 N-Ag J.

$\begin{array}{cc}\text { Berechnet für: } & \text { Gefanden: } \\ \mathrm{O}-\mathrm{CH}_{8}=4,61 & 4,5 \% \\ \mathrm{~N}-\mathrm{CH}_{3}=4,61 & 3,8 \%\end{array}$

Chelidonin: 0,2896 g Substanz gaben 0,1986 g N-dg J.

$\begin{array}{lc}\text { Berechnet für: } & \text { Gefunden: } \\ \mathrm{N}-\mathrm{CH}_{8}=4,0 & 3,8 \% \text {. }\end{array}$

4) Arch. d. Pharm. 241, 317-318. 
dort beobachtete Eigenschaft der Triboluminescenz mit dem hier beschriebenen Alkaloid aus Corydalis cava eine gewisse Aehnlichkeit aufweist. Da jedoch die bisher von ihm veröffentlichten Farbreaktionen eine große Verschiedenheit von den von mir bei dem Alkaloid $\mathrm{C}_{21} \mathrm{H}_{28} \mathrm{NO}_{7}$ festgestellten anfweisen uud Analysen noch nicht ausgeführt wurden, so ist eine Identitat beider Alkaloide nicht wahrscheinlich, jedenfalls aber jetzt noch nicht festzustellen.

Das bei $137,5^{\circ}$ schmelzende Alkaloid zeigt in seiner Zusammensetzung eine gewisse Aehnlichkeit mit dem bei $132^{\circ}$ schmelzenden Hydrastin; diesem kommt die Formel $\mathrm{C}_{21} \mathrm{H}_{21} \mathrm{NO}_{6}$ zu, jenem habe ich die Formel $\mathrm{C}_{21} \mathrm{H}_{28} \mathrm{NO}_{7}$ zugesprochen. Beide Alkaloide enthalten zwei $\mathrm{OCH}_{8}$-Gruppen und eine $\mathrm{NCH}_{8}$-Gruppe.

Auch für das Alkaloid $\mathrm{C}_{21} \mathrm{H}_{28} \mathrm{NO}_{7}$ habe ich aus den oben angeführten Gründen zur Zeit noch keinen Namen gewählt. Die Versuche werden fortgesetzt.

\section{Zusammenstellung der Resultate:}

1. Das Kraut von Corydalis cava enthält außer amorphen Basen von den bekannten Corydalisalkaloiden nur Bulbocapnin und zwei bisher nicht anfgefundene $\mathrm{C}_{21} \mathrm{H}_{21} \mathrm{NO}_{8}$ und $\mathrm{C}_{21} \mathrm{H}_{23} \mathrm{NO}_{7}$.

2. Protopin hat sich anch im Krante von Corydalis cava nicht auffinden lassen.

\section{B. Untersuchungen über die Konstitution des Corydalins.}

\section{Von Dr. Otto Haars.}

Das Corydalin gehört zu den wenigen Alkaloiden, deren Konstitution als ziemlich genan erforscht gelten kann. Freund und Josephi, ferner Ziegenbein hatten während der neunziger Jahre die Molekularformel zu $\mathrm{C}_{22} \mathrm{H}_{27} \mathrm{NO}_{4}$ endgültig festgelegt und dadarch bewiesen, daß frühere Autoren, die über das Corydalin gearbeitet hatten, stets Basengemische, nie aber das reine Corydalin analysiert hatten.

Wenn nun heute die Konstitution des Corydalins bereits als feststehend angenommen werden darf, so gebthrt das Verdienst der Erforschung der Strukturformel den englischen Chemikern Dobbie and Lauder ${ }^{1}$, die auf rein chemischem Wege ibr Ziel erreichten, andererseits J. Gad a mer ${ }^{2}$ ), der auf Grund physikalisch-chemischer Beobachtangen za denselben Schltissen gelangte wie Dobbie und

1) Transact. of the Chem. Soc. 1894 Corydaline Part III, 1897 Corydaline Part IV, 1899 Corydaline Part VI, 1902 Corydaline Part VII.

2) Ga da mer, Arch. d. Pharm. 240, 43 u. f. 